\title{
The Effect of Percutaneous Endovascular Aortic Repair on Aortic Dissection
}

\section{Cheng Chen}

Vascular Surgery, YUNNAN FUWAI CARDIOVASCULAR HOSPITAL, Kunming, Yunnan, 650000, China

\begin{abstract}
[Abstract] Objective: To explore the effects of percutaneous endo-aortic repair on patients with aortic dissection. Methods: Selected 100 patients with aortic dissection who were treated in Yunnan Fuwai Cardiovascular (YFC) Hospital from June 2020 to June 2021 as the research samples. The patients were divided into two groups, and different treatment methods were used. The percutaneous aorta was analyzed and compared. The clinical effect of endovascular repair was observed. Results: There were significant differences in hospitalization time, operation time and incidence of adverse reactions between the two groups of patients, expressed as $\mathrm{P}<0.05$. The incidence of adverse reactions in the experimental group was lower, the operation time and hospital stay were shorter, and the effect was better. Conclusion: The application of percutaneous aortic endovascular repair to the treatment of patients with aortic dissection has a significant clinical effect, which can shorten the patient's hospital stay, reduce the incidence of complications, and increase the degree of clinical attention.
\end{abstract}

Keywords: Percutaneous aortic endovascular repair; Aortic dissection; Incidence of adverse reactions

Publication date: May, 2021; Publication online: 31 May, 2021

*Corresponding author: Cheng Chen, 13578086160@sina.cn

\section{Introduction}

In clinical trials, the symptoms of aortic dissection are more common. This symptom belongs to a cardio-cerebrovascular disease. The patient has a more acute onset, more serious illness, greater damage to the patient, and higher clinical mortality. Therapeutic intervention needs to be clinically administered to the patient in a timely and effective manner [1]. The clinical effect of traditional treatment methods is not significant enough, the patient's condition control effect is poor, and the patient's mortality and disability rate are still high, based on the current development situation, which is influenced by factors such as the complexity of the patient's condition. Intra-wall repairs have emerged as a result of recent advancements in medical technology, with considerable clinical outcomes that have been promoted in clinics and have better treatment effects for patients ${ }^{[2]}$. This study is based on, selecting patients from YFC hospital as a sample group, to research on the implementation of percutaneous aortic endovascular repair, also compare and analyze its effect on patients.

\section{Data analysis and methods}

\subsection{General information}

Selected the patients in YFC hospital as a sample, where the selected 100 patients with aortic dissection received clinical trial treatment from June 2020 to June 2021 as a sample group, among them were 63 males and 37 females, the age range of the patients was 34-78 years old, and the average age of the patients was $(53.23 \pm 3.20)$ years old. The maximum diameter of the patient's aorta is $(4.90 \pm 0.53) \mathrm{cm}$, and the detection data of the true lumen is $(4.12 \pm 0.63) \mathrm{cm}$. The patients were divided into two groups where they were treated with different methods. The first group of 50 people were in the experimental group and the balance 50 people in the control group. Comparing the general data of the two groups of patients, the difference was small, $\mathrm{P}>0.05$, indicating significance. This study was carried out after the approval of the hospital ethics committee, and the patients were informed and they agreed to participate in this study. It can be ruled out that the patient does not have a history of mental illness and the possibility of incomplete data.

\subsection{Research methods}


In this study, both groups of patients were given endovascular aortic exclusion. In the specific implementation process, the actual situation of the patient needed to be grasped before the operation. Therefore, the CTA examination was used to clarify the blood supply of the patient's important organs, and the patient's dissecting rupture was observed, also a suitable vessel approach for the patient was chosen. Routine preoperative preparations was conducted where analgesic intervention, intravenous injection of sodium nitroprusside and esmolol, local anesthesia, and transmural puncture technology are done. When the puncture was successful, the $6 \mathrm{~F}$ radial artery expanded to enhance the sheath. The tube-like guide wire was inserted and inclined 45-50 degrees to the left and front to enhance the insertion of the double J-tube and performed thoracic aortic angiography.

The patients in the experimental group were again given disinfection treatment to the skin of the groin on both sides, and used aortic CTA to grasp the actual situation of the patient. Upon selecting the true lumen femoral artery as the punctured vessel, and selecting the proximal end of the femoral artery bifurcation for puncture, after the puncture intervention was completed, the suture device was sent into it and the patient was observed. When the patient's pulsatile bleeding was observed in the primary orifice of the patient, the suture device was placed. Then, the guide wire was exchanged, and another guide wire was placed in the $8 \mathrm{~F}$ arterial sheath. The suture device was used for suture processing.

The 50 patients in the control group were disinfected with bilateral groin skin, and anesthetized in the groin on the specified lumen side of the femoral artery, and were treated with arteriotomy.

\subsection{Observation indicators}

The hospitalization time, operation time and incidence of adverse reactions of the two groups of patients were compared. Adverse reactions mainly include poor wound healing and femoral artery thrombosis.

\subsection{Statistical methods}

For data statistics, SPSS20.0 software was mainly used. Among them, for the calculation of measurement data, the result was expressed mainly by $(\overline{\mathrm{X}} \pm \mathrm{s})$, the verification wad mainly based on the $t$ value, and the statistics of counting data, the comparison result adopts (n (\%)) for counting and verifying, it is expressed as a $\mathrm{X}^{2}$ value. When the result shows that the data is less than 0.05 hours, it means that the calculation of this study is significant.

\section{Results}

\subsection{Comparison of time indicators}

The two groups of patients have significant differences in the data of operation time and hospitalization time, $\mathrm{P}<0.05$, which is statistically significant, shown in Table $\mathbf{1 .}$

Table 1. Comparison of time indicators between the two groups of patients $(\overline{\mathrm{X}} \pm \mathrm{s})$

\begin{tabular}{ccc}
\hline Group & Operation time $(\mathrm{min})$ & Hospital stays $(\mathrm{d})$ \\
\hline Test group $(\mathrm{n}=50)$ & $85.45 \pm 10.23$ & $5.38 \pm 1.92$ \\
Control group $(\mathrm{n}=50)$ & $131.24 \pm 34.52$ & $8.75 \pm 2.12$ \\
$\mathrm{t}$ & 10.294 & 7.596 \\
$\mathrm{P}$ & $<0.05$ & $<0.05$ \\
\hline
\end{tabular}

\subsection{The incidence of adverse reactions}

reactions between the two groups of patients, $\mathrm{P}<0.05$, which

There is a significant difference in the incidence of adverse is statistically significant, see Table 2.

Table 2. Comparison of the incidence of adverse reactions between the two groups of patients [n(\%)]

\begin{tabular}{cccc}
\hline Group & Poor wound healing & Femoral artery thrombosis & Incidence \\
\hline Test group $(\mathrm{n}=50)$ & $1(2.00)$ & $1(2.00)$ & $2(4.00)$ \\
Control group $(\mathrm{n}=50)$ & $3(6.00)$ & $3(6.00)$ & $6(12.00)$ \\
$\mathrm{X}^{2}$ & & & 5.403
\end{tabular}




\section{Discussion}

Aortic dissection poses a greater threat to the patient's health, and its incidence is high. It is a cardio-cerebrovascular disease. The patient not only has a more acute onset, but also has a more serious condition, which is more damaging to the patient's health and has a higher clinical mortality rate. There is a crucial need for clinical treatment to patients in immediately. There are multiple techniques to treat patients, based on the current state of development. Medical and surgical treatment are the most common traditional treatments. The two strategies have an average therapy effect, making it difficult to effectively enhance patient recovery. The incidence of postoperative complications is relatively high. The patient has a longer recovery time, and it is difficult to effectively promote it in clinical trials ${ }^{[3]}$. Under normal circumstances, medical treatment is based on the patient's actual situation; giving patients targeted drug intervention is only a basic treatment that can control the patient's condition but is difficult to completely cure; surgical treatment is primarily based on aortic resection and artificial blood vessel replacement. The application effect of this method is more significant than that of drug treatment, but it causes more trauma to the patient, and the incidence of postoperative complications is higher. The operation time of the method is longer, and the risk on the patient is higher during the operation. Therefore, it is difficult to ensure the effective recovery of the patient ${ }^{[4]}$.

With the gradual advancement of clinical development, endovascular repair has emerged, and its application in aortic dissection is different from surgical treatment approach ${ }^{[5]}$. After the application of this technology in this study, the results showed that the experimental group of patients had shorter operation time and hospital stay, and the incidence of adverse reactions was lesser. The data difference between the two groups of patients was vast, indicating that the percutaneous aortic cavity Prosthetic surgery is more effective for patients. The implementation of this technology is easy to operate, can effectively shorten the operation time, reduce the patient's surgical risk, guarantee the patient's life safety, promote the surgical effect, and improve the patient's quality of life. At the same time, this technology is less traumatic to patients. It can reduce the incidence of complications for patients and is of positive significance for patient recovery. It can improve patient's clinical symptoms and reduce their pain. After the operation, the medical staff can start from the present condition of the patient and give the patient a combination of different drugs, such as antiplatelet drugs, which can maintain the effect of the operation on the patient and promote the recovery of the patient. The clinical application is significant ${ }^{[6]}$.

\section{Conclusion}

In summary, the application of percutaneous endovascular aortic repair in the treatment of patients with aortic dissection has significant clinical effects, which can shorten the patient's hospital stay, reduce the incidence of complications, increase clinical attention and actively promote its implementation.

\section{References}

[1] Li WR, Li QL, Zhang XM, et al., 2019, Mid and Long-Term Results of Chimney Technique in Endovascular Repair of Abdominal Aortic Aneurysm, Chinese Journal of General Surgery. 34(3):222-224.

[2] Lin T., 2019, Meta-Analysis of the Long-Term Effects of Endovascular Repair and Open Surgery for the Treatment of Abdominal Aortic Aneurysm, Fujian Medical University.

[3] Wang CS, Zhu PX, Li JJ, et al., 2020, Clinical Efficacy Analysis of 70 Cases of Sub-Renal Abdominal Aortic Aneurysm Treated with Endovascular Repair, Modern Diagnosis and Treatment. 31(17):2753-2754.

[4] Yong H, Ge XH., 2019, Experience in the Treatment of Complications Related to Endovascular Repair of Abdominal Aortic Aneurysm, World Clinic Medicine. 13(004):71-73.

[5] Li XC, Chen YH, Zhao LM, et al., 2019, Application of Modified Puncture and Suture Technique in Endovascular Repair of Abdominal Aortic Aneurysm, Chinese Journal of Vascular Surgery (Electronic Edition), (4).

[6] Pang HJ., 2019, Morphological Observation of Aortic Remodeling after Endovascular Repair of Complex Acute Type B Aortic Dissection and its Effect on the Long-Term Survival of Patients, Southern Medical University. 